\title{
CONFLICT-FREE VERTEX-CONNECTIONS OF GRAPHS
}

\author{
Xueliang Li ${ }^{1}$, Yingying Zhang, Xiaoyu Zhu \\ Center for Combinatorics and LPMC \\ Nankai University \\ Tianjin 300071, China \\ e-mail: lxl@nankai.edu.cn \\ zyydlwyx@163.com \\ zhuxy@mail.nankai.edu.cn \\ YAPING MaO, Haixing ZhaO \\ School of Mathematics and Statistics \\ Qinghai Normal University \\ Xining, Qinghai 810008, China \\ e-mail: maoyaping@ymail.com \\ h.x.zhao@163.com \\ AND \\ STANISLAV JENDROL' \\ Institute of Mathematics \\ P.J. Šafárik University \\ Jesenná 5, 04001 Košice, Slovakia \\ e-mail: stanislav.jendrol@upjs.sk
}

\begin{abstract}
A path in a vertex-colored graph is called conflict-free if there is a color used on exactly one of its vertices. A vertex-colored graph is said to be conflict-free vertex-connected if any two vertices of the graph are connected by a conflict-free path. This paper investigates the question: for a connected graph $G$, what is the smallest number of colors needed in a vertex-coloring of $G$ in order to make $G$ conflict-free vertex-connected. As a result, we get that the answer is easy for 2-connected graphs, and very difficult for connected graphs with more cut-vertices, including trees.
\end{abstract}

Keywords: vertex-coloring, conflict-free vertex-connection, 2-connected graph, tree.

2010 Mathematics Subject Classification: 05C15, 05C40, 05C75.

\footnotetext{
${ }^{1}$ Corresponding author.
} 


\section{REFERENCES}

[1] J.A. Bondy and U.S.R. Murty, Graph Theory (Springer, 2008).

[2] H. Chang, Z. Huang, X. Li, Y. Mao and H. Zhao, Nordhaus-Gaddum-type theorem for conflict-free connection number of graphs. arXiv:1705.08316 [math.CO].

[3] H. Chang, M. Ji, X. Li and J. Zhang, Conflict-free connection of trees. arXiv:1712.10010 [math.CO]

[4] G. Chartrand, G.L. Johns, K.A. McKeon and P. Zhang, Rainbow connection in graphs, Math. Bohem. 133 (2018) 85-98.

[5] G. Chartrand, G.L. Johns, K.A. McKeon and P. Zhang, The rainbow connectivity of a graph, Networks 54 (2009) 75-81.

doi:10.1002/net.20296

[6] L. Chen, X. Li and Y. Shi, The complexity of determining the rainbow vertexconnection of a graph, Theoret. Comput. Sci. 412 (2011) 4531-4535.

doi:10.1016/j.tcs.2011.04.032

[7] J. Czap, S. Jendrol' and J. Valiska, Conflict-free connections of graphs, Discuss. Math. Graph Theory 38 (2018) 911-920.

doi:10.7151/dmgt.2036

[8] B. Deng, W. Li, X. Li, Y. Mao and H. Zhao, Conflict-free connection numbers of line graphs, Lecture Notes in Comput. Sci. 10627 (2017) 141-151. doi:10.1007/978-3-319-71150-8_14

[9] A.V. Iyer, H.D. Ratliff and G. Vijayan, Optimal node ranking of trees, Inform. Process. Lett. 28 (1988) 225-229. doi:10.1016/0020-0190(88)90194-9

[10] M. Krivelevich and R. Yuster, The rainbow connection of a graph is (at most) reciprocal to its minimum degree, J. Graph Theory 63 (2010) 185-191.

[11] X. Li and S. Liu, Tight upper bound of the rainbow vertex-connection number for 2-connected graphs, Discrete Appl. Math. 173 (2014) 62-69. doi:10.1016/j.dam.2014.04.002

[12] X. Li, Y. Mao and Y. Shi, The strong rainbow vertex-connection of graphs, Util. Math. 93 (2014) 213-223.

[13] X. Li and Y. Shi, On the rainbow vertex-connection, Discuss. Math. Graph Theory 33 (2013) 307-313. doi:10.7151/dmgt.1664

[14] X. Li, Y. Shi and Y. Sun, Rainbow connections of graphs: A survey, Graphs Combin. 29 (2013) $1-38$. doi:10.1007/s00373-012-1243-2

[15] X. Li and Y. Sun, Rainbow Connections of Graphs (Springer, New York, 2012). doi:10.1007/978-1-4614-3119-0 
[16] Z. Li and B. Wu, On the maximum value of conflict-free vertex-connection number of graphs. arxiv: 1709.01225 [math.CO]

[17] H. Liu, Â. Mestre and T. Sousa, Rainbow vertex $k$-connection in graphs, Discrete Appl. Math. 161 (2013) 2549-2555.

doi:10.1016/j.dam.2013.04.025

Received 22 May 2017

Revised 18 January 2018

Accepted 22 January 2018 